\title{
HREM and Analytical TEM of the Rapidly Solidified Microstructure Evolved at the Extremes of the Coupled Crystal Growth Regime in Hypo-Eutectic Al-Cu Alloys
}

\author{
Vishwanadh Bathula $^{1,2}$, Subarna Khanal ${ }^{1}$, and Jörg M.K. Wiezorek ${ }^{1}$ \\ 1. Dept. of Mechanical Engineering and Materials Science, University of Pittsburgh, Pittsburgh, PA, \\ USA \\ 2. Materials Science Division, Bhabha Atomic Research Centre, Mumbai, India.
}

Non-equilibrium cooling induces metastable phases and atypical microstructures in multi-component alloys during laser-melting facilitated welding and additive manufacturing [1,2]. Prior work showed that laser induced melting of electron-transparent alloy films results in solidification microstructures morphologically identical to those in bulk equivalents [2,3]. In situ imaging of the melt-pool size evolution with nanoscale spatio-temporal resolution using the Dynamic Transmission Electron Microscope (DTEM) permitted determination of the solidification interface velocity evolution and the critical conditions under which composition dependent crystal growth mode changes occur in hypoeutectic Al-Cu alloys ( $\sim 3$ at. $\%$ $\mathrm{Cu}$ ) [2,3]. The rapid solidification microstructures in these Al-Cu alloys exhibit characteristically four morphologically distinct zones that form as the solidification interface accelerates and is driven ever farther away from equilibrium: 1. heat affected zone (HAZ); 2. transition zone; 3. columnar growth zone; 4. banded region (Fig. 1 (a)). In all four zones, in addition to the primary $\alpha$-Al phase, presence of second phase has been observed. Analyses of selected area electron diffraction patterns and precession electron diffraction based phase mapping [3] revealed $\mathrm{Al}_{2} \mathrm{Cu} \theta$-phase in the $\mathrm{HAZ}$ and $\mathrm{Al}_{2} \mathrm{Cu} \theta$ '-phase in the remaining three regions. The two-phase regions of the columnar zone and the banded morphology grains ( 3 and 4 in Fig. 1) formed by coupled growth of $\alpha-\mathrm{Al}$ and $\theta^{\prime}-\mathrm{Al}_{2} \mathrm{Cu}$ crystals and exhibit discontinuous distributions of the later within the former. Precession electron diffraction-based phase and orientation mapping showed that the $\theta^{\prime}-\mathrm{Al}_{2} \mathrm{Cu}$ establishes a cube-on-cube orientation relationship (OR) with the surrounding $\mathrm{Al}$ phase [3], which minimizes interfacial energy and is consistent with OR's reported in solidi-state precipitation [4]. HREM images of the Al matrix and $\theta^{\prime}$ phase acquired along [001] zone axis (e.g. Fig.1(b)) reveal coherent interface sections, which impart a kinetic advantage to the $\theta^{\prime}-\mathrm{Al}_{2} \mathrm{Cu}$ over the thermodynamically favored $\theta-\mathrm{Al}_{2} \mathrm{Cu}$ during the required nucleation stage prior to subsequent growth into the liquid responsible for establishing the discontinuous $\theta^{\prime}$-phase distributions in the $\alpha$-phase grains. The spheroidal morphology of the $\theta$ '-phase observed here differs from the plate-shape characteristic of solid-state transformations [4] and is consistent with the growth taking place into the liquid. The banded region forms when the solidification interface reaches the composition dependent critical velocity for complete solute trapping, which triggers a transition from coupled crystal growth in zone 3 to single-phase partitionless solidification [2,3]. The banded region comprises single crystal grains of the $\alpha$-Al phase with periodically altered morphology of partitionless solidified single-phase bands with on average alloy composition and two-phase bands (e.g. marker A-E, Fig. 1(a)). An example STEM image and the corresponding energy-dispersive X-ray spectroscopy (EDXS) based composition map of the transition from the columnar zone to the banded region for Al-11 at $\% \mathrm{Cu}$ are shown in Fig.1(c), (d), respectively. The EDXS mapping showed supersaturated $\alpha$-Al in the coupled growth regions containing on average $\sim 8$ $9 \mathrm{at} \% \mathrm{Cu}$, and $11.5 \mathrm{at} \% \mathrm{Cu}$, the alloy composition, in the single-phase growth bands of the banded region, which significantly exceed the solubility limit, $\sim 2.6 \mathrm{at} \% \mathrm{Cu}$. The single-phase bands of the banded region (Fig.1(e)) show spatial modulations of the $\mathrm{Cu} \%$ that significantly deviate from the alloy composition with spatial frequency that correlates well to the scale of $\theta^{\prime}-\mathrm{Al}_{2} \mathrm{Cu}$ phase in the coupled growth regions. We 
posit that these composition modulations give evidence to the presence of $\theta$ '-phase 'embryos' in the $\alpha$-Al single phase bands, which formed by partitionless single-phase crystal growth [5].

\section{References:}

[1] J. Kline and J. Leonard, Applied physics letters 86 (2005), p. 201902.

[2] J.T. McKeown, et al, Acta Materialia 65 (2014), p. 56.

[3] K.W. Zweiacker, et al, Acta Materialia 145 (2018), p. 71.

[4] S.C. Wang and M.J. Starink, International Materials Reviews 50 (2005), p. 193.

[5] This work received support from the National Science Foundation, NSF-1607922.
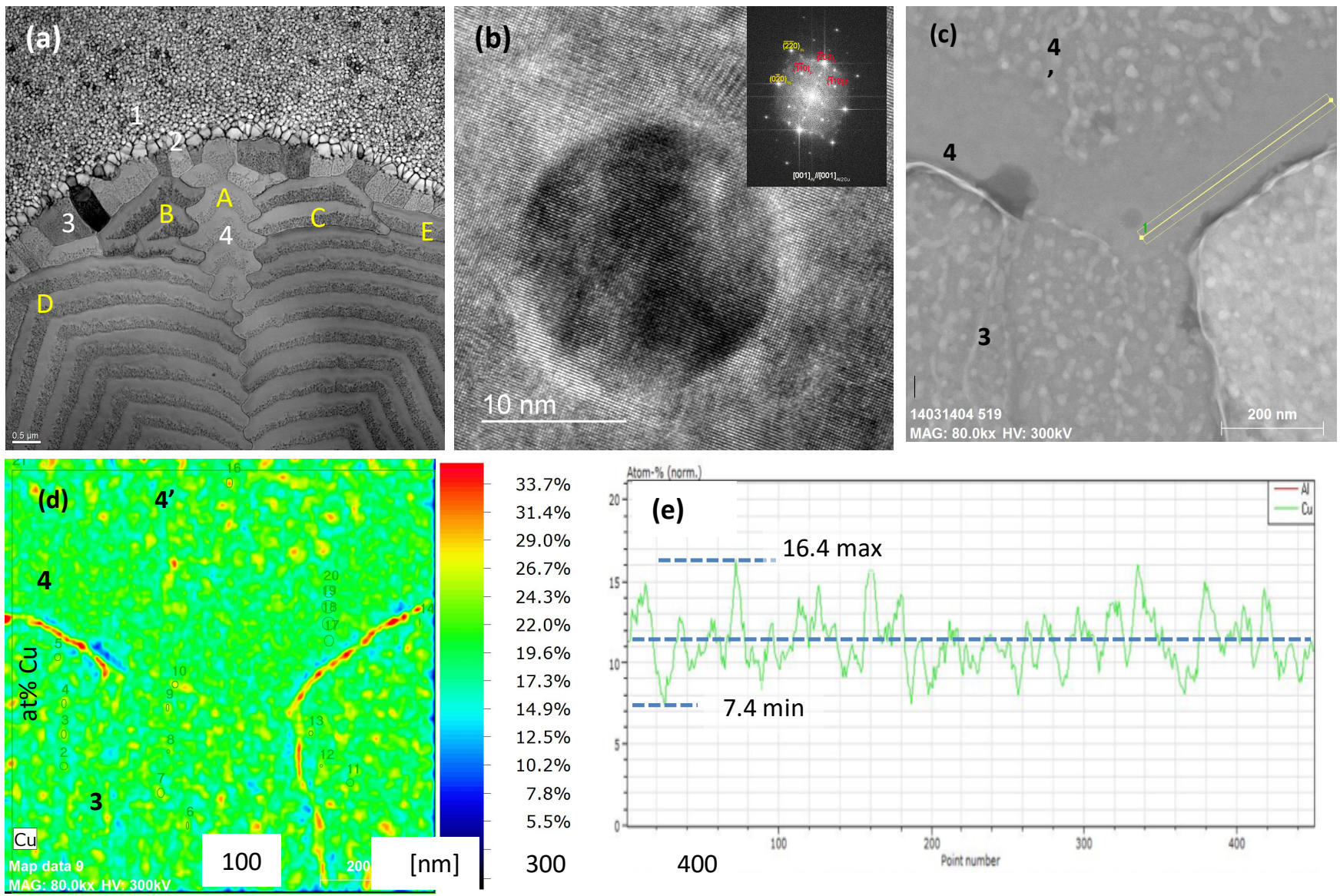

Figure 1. (a) STEM (scale marker $500 \mathrm{~nm}$ ) of melt pool in $\mathrm{Al}-11 \mathrm{at} \% \mathrm{Cu}$ with four distinct microstructure zones marked as 1, 2, 3 and 4; four banded morphology grains marked as A, B, C, D, E. (b) HREM of $\theta^{\prime}$ $\mathrm{Al}_{2} \mathrm{Cu}$ and $\alpha-\mathrm{Al}(\mathrm{Cu})$ in the coupled growth band in zone 4, [001] zone axes. (c) HAADF STEM (200 nm scale marker) of transition from coupled growth in zone 3 (marker 3) to single-phase growth of $\alpha$-Al in partitionless band (marker 4) and back to coupled growth in partioned band (marker 4'). (d) Corresponding STEM EDXS Cu map of area in (c). (e) $\mathrm{Cu} \%$ modulation along trace in (c), $1 \mathrm{~nm}$ steps. 